\title{
Multifocal eosinophilic granuloma presenting as progressive brainstem and cerebellar dysfunction
}

\author{
Toshiyuki Fukazawa, Tetsuro Yanagihara, Koji Hamada, Takeshi Hamada
}

\begin{abstract}
A 55 year old woman with multifocal eosinophilic granuloma (MEG) is described. She developed facial numbness and twitching followed by slowly progressive cerebellar symptoms. Two years later polyuria and polydipsia were noted. A CT of the brain showed multifocal enhancing lesions, and MRI showed areas of hyperintensity on $\mathrm{T} 2$ weighted studies in the cerebellar peduncles, pons, and midbrain. Radiographs of the skull, pelvis, and long bones were normal, but a ${ }^{99 m}$ Tc diphosphonate bone scan and MRI showed bone lesions compatible with granuloma. The diagnosis of MEG was made by bone biopsy. This is believed to be the first case of MEG with such unusual clinical profiles and radiographical findings. Skeletal surveys are indicated for patients with unexplained focal or multifocal inflammatory changes in the cerebellum.
\end{abstract}

$(\Im$ Neurol Neurosurg Psychiatry 1994;57:980-982)

Multifocal eosinophilic granuloma (MEG; Hand-Schüller-Christian disease), usually diagnosed in children and young adults, may have CNS manifestations. This is probably due to infiltration of abnormal histiocytes into the hypothalamus or posterior pituitary gland with resultant diabetes insipidus and other endocrine abnormalities. The most common site for extradiencephalic CNS involvement seems to be the cerebellum. ${ }^{1-3}$ Endocrine disorders or peripheral bone lesions mostly develop early and the diagnosis of MEG is usually made before ataxia develops. ${ }^{1-4}$ Our patient presented with brainstem and cerebellar dysfunction in middle age two years before the onset of diabetes insipidus. Furthermore, CT showed extremely unusual findings of multifocal enhancing lesions, ${ }^{1-3}$ suggestive of a metastatic brain tumour, and MRI suggested an inflammatory process. A ${ }^{99 m}$ TC diphosphonate scan and MRI showed bone lesions, leading to the diagnosis of MEG by bone biopsy, but with normal plain radiographs. The importance of skeletal surveys for patients with slowly progressive cerebellar and brainstem dysfunctions is discussed.
Case report

A 55 year old woman was in good health until February 1990, when she noticed left facial numbness. Neurological examination was normal except for subjective left facial sensory disturbance. In June 1990 she developed an unsteady gait and dysarthria. In April 1991 she began to fall easily, and in September 1991 she was diagnosed with spinocerebellar degeneration at another hospital. In June 1992 she developed a pronounced thirst, increased water intake, and increased urinary output. The ataxia and dysarthria worsened and she was admitted to Hokuyukai Neurology Hospital on 10 September 1992.

Examination showed a well nourished woman with no exophthalmos and no bony tenderness. She had severe thirst, drinking 5 to 8 litres of water in a 24 hour period, accompanied by excessive urination. Rightward gaze evoked nystagmus and slight upbeat nystagmus during upward gaze was present as well as a cerebellar dysarthria. A left hemifacial spasm and a left peripheral facial paresis were found, with a mild atrophy of the left masseter and hypesthesia in the second division of the left trigeminal nerve. Otherwise, sensory examination gave normal results. Strength was normal, but decreased muscle tone and bilateral Babinski's signs were present. Dysmetria in all limbs was evident, especially in the legs. This was more prominent on the left side, and there was severe gait ataxia.

Laboratory tests of adrenal, thyroid, renal, hepatic, and haematological functions, including alkaline phosphatase, gave normal results. She had negative serological evaluations for syphilis, sarcoidosis, and autoimmune diseases. A skin test for tuberculosis was negative. The presence of diabetes insipidus with a satisfactory response to vasopressin was established by a water deprivation test. Examination of CSF showed it to be normal, without an increase of IgG concentration and IgG index.

CT showed multiple abnormal enhancing lesions accompanied by poorly demarcated low density areas (fig 1), and MRI of the brain showed multifocal irregular areas of hyperintensity on T2 weighted studies (fig 2), in the medial portions of the cerebellar hemisphere, cerebellar peduncles, midbrain, and pons. Cerebellar dysfunction with diabetes 
Figure 1 CT with contrast material showing multifocal enhancing cerebellar hemisphere, cerebellar peduncles, and pons. lesions in the midline of the

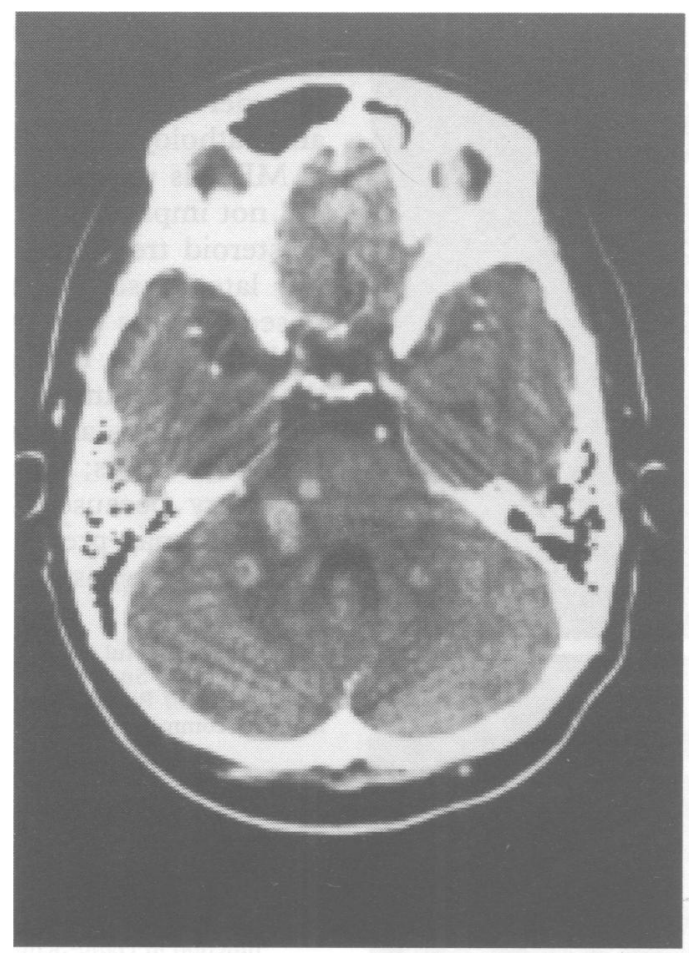

insipidus led us to be concerned about a histiocytosis and we performed a series of skeletal surveys. A ${ }^{99 \mathrm{~m}} \mathrm{Tc}$ diphosphonate bone scan showed areas of increased uptake in the right femur, and in the pelvis, whereas plain radiographs of the chest, skull, long bones, and pelvic bones were normal. A bone MRI with gadolinium contrast material (GdDTPA) showed abnormal enhancing lesions in the right femur and left acetabulum, and a bone biopsy of the acetabular lesion was performed. Histological examination disclosed a diffuse hypercellularity due to an infiltration of histiocytes, lymphocytes, and eosinophils in a fibrotic bed; a storiform pattern was not present (fig 3). Histiocytic cells were stained

Figure 2 Axial T2 weighted MRI $(T R=3000$, $T E=80)$ showing multifocal irregular areas of hyperintensity in the cerebellar hemisphere, cerebellar peduncles, pons, and midbrain.

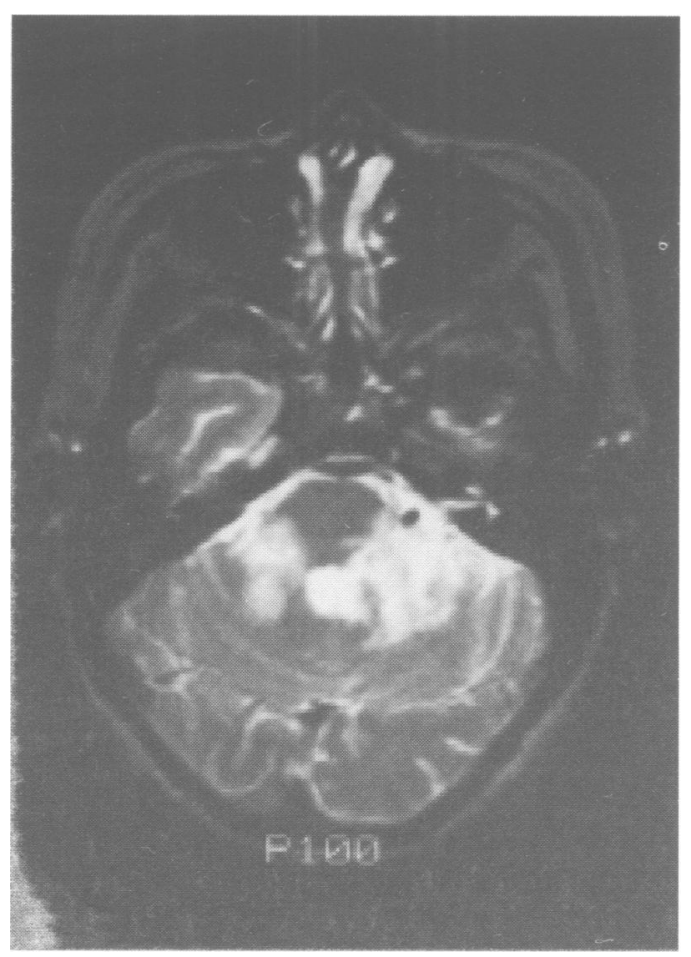

positive by anti-S100 protein, forming nests of the cells. These histological findings were compatible with eosinophilic granuloma.

Intravenous methylpredonisolone $(1.0 \mathrm{~g} /$ day $)$ for three days followed by a tapering course of oral predonisolone, and radiation therapy were given, with no effect on the clinical features or CT findings. After the radiation therapy the patient's general condition deteriorated, she developed spasticity of the legs, and was confined to bed afterwards.

\section{Discussion}

Our patient had histologically confirmed evidence of eosinophilic granuloma in the bone. CT findings for the brain mimicked those of metastatic tumours, but this could be ruled out by her clinical course of over three years. Results of serial CT studies after treatment with high doses of methylpredonisolone and normal IgG profiles of the CSF did not support a demyelinating process. The other differential diagnoses included tuberculosis, mycotic disease, syphilis, and sarcoidosis, but they were unlikely because of a lack of any evidence during the 10 month follow up. We believe that she had the same pathological process in the cerebellum and brainstem as in the bones.

MEG is most often a disease of childhood, but the disease in our patient seemed to begin in middle age. Lesions of the CNS are usually limited to the hypothalamus and posterior pituitary gland, although cerebellar manifestations may occur. ${ }^{1-5}$ Furthermore, it is unusual for MEG to present with these extradiencephalic involvements unless diabetes insipidus is demonstrable, and in most of the patients described with these abnormalities, peripheral bone lesions or diabetes insipidus developed early and the diagnosis of MEG had been made before ataxia was noted. ${ }^{1-5}$ Our patient had progressive cerebellar and brainstem symptoms two years before the onset of diabetes insipidus. She had no appearance of bony lesions characteristic of eosinophilic granuloma on plain radiographs, whereas a bone scan by an additional MRI study with Gd-DTPA showed the lesions compatible with granulomas. These bony lesions were probably too early to be seen on plain radiographs. Any other clue to the diagnosis, such as exophthalmos, was absent. Previous reports indicated that the CT abnormalities in the patients with MEG and cerebellar signs were of three types ${ }^{1-3}:$ (a) a decrease in cerebellar parenchymal absorption coefficient; $(b)$ a bilateral calcification in the dentate nucleus; $(c)$ a dilatation of the fourth ventricle and of the subarachnoid cisterns in the midline of the posterior fossa. Our patient had multifocal abnormal enhancing lesions with surrounding irregular low density areas, mimicking metastatic tumours. Our patient is, to our knowledge, the first case of MEG with such abnormalities in the cerebellum shown on CT. This seems to correlate with the known histopathological changes of histiocytic granuloma. ${ }^{5}$ 
Figure 3 (A) Lesion of the left acetabulum showing nests of pleomorphic cells in a fibrotic bed (haematoxylin-eosin; originally $\times 50$ ). (B) High magnification showing the lesion composed of histiocytes and eosinophils intermingled with scattered lymphocytes (haematoxylin - eosin; originally $\times 200$ ).

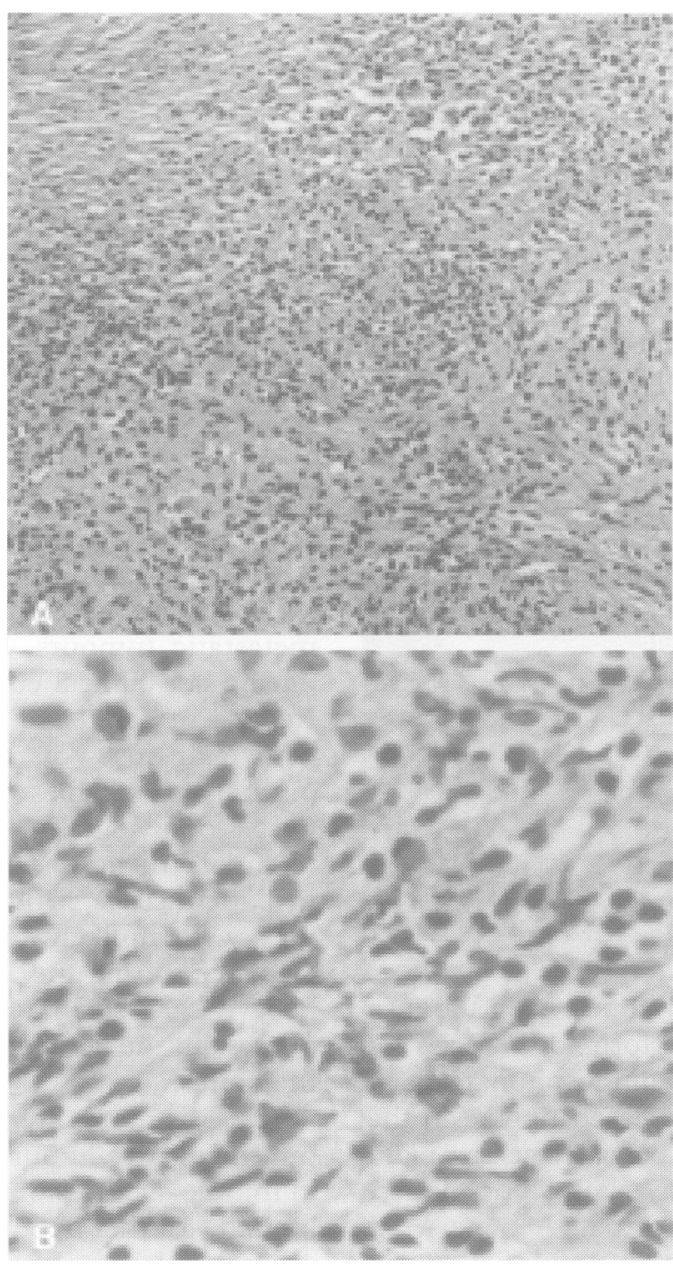

Figarella-Branger et $a l^{6}$ reported a primary histiocytic tumour of the cerebellum with pathological features similar to those in our patient. There was no description of skeletal surveys, however, and the authors concluded that "atypical inflammatory histiocytic tumour of the cerebellum" best characterised the lesion after craniotomy. Smith et $a l^{7}$ reported a patient with systemic histiocytosis presenting as multiple sclerosis with a clinical course and radiological findings similar to those in our patient; then Erdheim-Chester disease, another systemic histiocytosis, was diagnosed by brain biopsy with further skeletal evaluations. Our patient did not have this disorder because of different radiographical and pathological findings in the bones. ${ }^{78}$

MEG is potentially treatable. ${ }^{9}$ Our patient did not improve after either radiation therapy or steroid treatment, but it might have been too late to start treatment. ${ }^{10}$ Thus undiagnosed patients with cerebellar signs should be evaluated with radiographs of the skull and the pelvis, commonly involved sites of MEG. A bone scan is also indicated, as it can identify early lesions; repeated evaluations may be necessary because CNS signs and diabetes insipidus may precede the bone involvement by many years, as in our patient.

We are very grateful to Dr Naoki Takeda of the Department of Orthopaedic Surgery and to Dr Takayuki Nojima of the Department of Pathology, Hokkaido University, for valuable help and comments.

1 Braunstein GD, Whitaker JN, Kohler PO. Cerebellar dysfunction in Hand-Schuller-Christian disease. Arch Intern Med 1973;132:387-90.

2 Adornato BT, Eil C, Head GL, Loriaux DL. Cerebellar involvement in multifocal eosinophilic granuloma: demonstration by computed tomographic scanning. Ann Neurol 1980;7:125-9.

3 Birnbaum DC, Shields WD, Lippe B, Perlman S, Phillipart M. Idiopathic central diabetes inspidus followed by progressive spastic cerebellar ataxia. Arch Neurol 1989;46:1001-3.

4 Pomarède $R$, Czernichow $P$, Rappaport $R$, Royer P. Le diabète inspide pitresso-sensible de l'enfant. II. Etude diabete inspide pitresso-sensible de l'enfant. II. Etude de 93 cas obser

5 Beard W, Foster B, Kepes JJ, Guillan RA. Xanthmatosis of the central nervous system. Clinical and pathological observations of a case with a posterior fossa syndrome. Neurology 1970;20:305-14.

6 Figarella-Branger D, Gambarelli D, Perez-Castillo M, Regis J, Peragut JC, Pellissier JF. Atypical inflammatory histiocytic tumor of the cerebellum. A histological immunohistochemical, and ultrastructural study. $A m \mathscr{F}$ Surg Pathol 1990;14:778-83.

7 Smith ME, Katz DA, Harris JO, Frank JA, Kufta CV, McFarlin DE. Systemic histiocytosis presenting as multiple sclerosis. Ann Neurol 1993;33:549-54.

8 Miller RL, Sheeler LR, Bauer TW, Bukowski RM Erdheim-Chester disease. Case report and review of the literature. Am $\mathcal{F}$ Med 1993;80:1230-6.

9 Zinkham WH. Multifocal eosinophilic granuloma. Natural history, etiology and management. $\mathrm{Am} \mathcal{J} \mathrm{Med}$

10 Smith DG, Nesbit ME Jr, D'Angio GJ, Levitt SH. Histiocytosis X: Role of radiation therapy in management with special reference to dose levels employed. Radiology 1973;106:419-22. 